\title{
ASOCIACIÓN ENTRE EL CONSUMO DE CIGARRILLO Y ALCOHOL EN LA GESTANTE COMO FACTOR DE RIESGO PARA LABIO Y|O PALADAR HENDIDO NO SINDRÓMICO
}

\author{
${ }^{1}$ Ethman Ariel Torres Murillo, ${ }^{2}$ German Gómez Lázaro, ${ }^{2}$ Zonia Pinzón Bayona \\ ${ }^{1}$ Odontólogo U. Santo Tomás, Especialista en Estomatología Pediátrica U. Nacional de Colombia, Especialista en Ortodoncia U. Santo Tomás, \\ Docente U. Santo Tomás, Colombia. \\ ${ }^{2}$ Odontólogo U. Santo Tomás, Especialista en Ortodoncia U. Santo Tomás, Colombia.
}

Autor responsable de correspondencia: Ethman Ariel Torres Murillo

Correo electrónico: ethmant@yahoo.com

\section{RESUMEN}

Objetivo: determinar la asociación entre el consumo de cigarrillo y alcohol en la mujer embarazada como factor de riesgo para labio y/o paladar hendido no sindrómico e hipodoncia.

Materiales y métodos: se realizó un estudio de corte trasversal con una muestra de 79 pacientes entre los 4 y 18 años que habían sido diagnosticados con labio y/o paladar hendido no sindrómico. Se realizó una encuesta a las madres, se diligenció el formulario del Estudio Colaborativo Latinoamericano de Malformaciones Congénitas (ECLAM) y se tomó una radiografía panorámica a los pacientes. Se realizó un análisis descriptivo, se usó la prueba de $\mathrm{Chi}^{2}$ y Test exacto de Fisher para las variables cualitativas y t de Student o U. de Mann Whitney para las cuantitativas. Una regresión logística determinó la asociación entre las variables consumo de cigarrillo y alcohol con la presencia de labio y/o paladar hendido no sindrómico. Se consideró un valor alpha igual a 0,05.

Resultados: el 96\% de la población estudiada presentó labio y/o paladar hendido, relacionándose con el lado en que el paciente tenía la hipodoncia, que se presentó en el 85\% de la muestra. En los pacientes con labio y paladar hendido se encontró hipodoncia en el $85 \%$ de los casos, la hipodoncia del incisivo lateral superior es la más frecuente en el $81 \%$ de los casos, seguido de la hipodoncia de premolares, en el 7\% de los casos. El 35\% de las madres presentó antecedente de fumadora y el $43 \%$ acostumbraba a tomar alcohol. Se encontró que las mujeres en embarazo que habían consumido alcohol tenían una probabilidad 1,5 veces de tener un hijo/a con labio y/o paladar hendido aunque este valor no fue estadísticamente significativo [OR 1,5 IC 95\% 0,13-17,66].

Conclusiones: al determinar la asociación entre el consumo de cigarrillo y alcohol como factor de riesgo en labio y paladar hendido, no se encontró una relación estadísticamente significativa, pero sí una relación positiva entre las variables mencionadas. [Torres EA, Gómez G, Pinzón Z. Asociación entre el consumo de cigarrillo y alcohol en la gestante como factor de riesgo para labio y/o paladar hendido no sindrómico. Ustasalud 2012; 11: 88 - 94]

Palabras clave: Labio leporino, Hábito de fumar, Anodoncia

\section{ASSOCIATION BETWEEN CIGARETTE AND ALCOHOL CONSUMPTION IN PREGNANT AS A RISK FACTOR FOR NON-SYNDROMIC CLEFT LIP AND/OR PALATE}

\section{ABSTRACT}

Objective: to assess the association between cigarette smoking and alcohol consumption in pregnant women as a risk factor for cleft lip and / or non-syndromic cleft palate and hypodontia.

Methods: a cross-sectional study was done with a sample of 79 patients between 4 and 18 years who had been diagnosed with nonsyndromic cleft lip and/or cleft palate. We conducted a survey of mothers, the form from Latin American Collaborative Study of Congenital Malformations (ECLAM) was filled out and a panoramic x-ray was taken for every patient. We performed a descriptive analysis, we used the $\mathrm{Chi}^{2}$ test and Fisher exact test for qualitative variables and Student t test or U. Mann Whitney test for quantitative variables. A logistic regression determined the association between the variables smoking and alcohol consumption in the presence of lip and / or non-syndromic cleft palate. An alpha value equal to 0.05 was considered.

Results: $96 \%$ of the studied population had lip and/or cleft palate, interacting with the side on which the patient had hypodontia, which was present in $85 \%$ of the sample. Hypodontia was found in $85 \%$ of the patients with cleft lip, with the upper lateral incisor hypodontia most prevalent in $81 \%$ of cases, followed by hypodontia of premolars in $7 \%$ of the cases. The $35 \%$ of the mothers presented a history of smoking and $43 \%$ used to drink alcohol. It was found that pregnant women who consumed alcohol were 1.5 times likely to have a son / daughter with cleft lip and/or palate although this value was not statistically significant [OR $1.5,95 \%$ CI 0.13 - 17.66 ]. Conclusions: there was no statistically significant value to determine the association between cigarette smoking and alcohol as a risk factor for cleft lip and palate but a positive relationship between these variables was found.

Key words: Orofacial cleft, Smoking, Anodontia, Risk factor 


\section{INTRODUCCIÓN}

El labio y paladar hendido es la malformación craneofacial más frecuente y una de las más estudiadas y menos entendidas por presentar una etiología diversa con múltiples modelos fisiopatológicos. Padecerla ocasiona alteraciones esqueléticas, dentales, funcionales y estéticas que traen importantes repercusiones en la vida de los pacientes y sus familias. Además, es un tema relevante para los equipos de salud que atienden a estas personas, al ser considerados pacientes complejos por los elevados costos de su atención y la cantidad de procedimientos que se necesitan para lograr su rehabilitación integral.

La prevalencia del labio y paladar hendido varía de acuerdo con la zona geográfica y raza estudiada. En Colombia se ha reportado desde 1 en 500 a 1 en 1000 nacidos vivos; ${ }^{1}$ para Bucaramanga en el Hospital Universitario de referencia se encontró una prevalencia de 1,56 por mil nacidos vivos durante el período 2005 a $2009 .^{2}$

La etiología del labio y paladar hendido es poligénica y multifactorial. ${ }^{3}$ La parte genética ha sido estudiada en el ámbito mundial y se conoce que las alteraciones entre epitelio y mesénquima son las más importantes, pero que existen dos grupos de genes que regulan dichas interacciones, estos son los factores de transcripción (homeobox) y genes implicados en vías de señalización (factores de crecimiento) ${ }^{4,5}$ y que estas alteraciones son la causa de padecer la hendidura que se desarrolla embriológicamente entre la IV a la XII semana de gestación. ${ }^{6,7}$ Los estudios genéticos se han realizado en diferentes poblaciones como la chilena ${ }^{8}$ y colombiana ${ }^{9}$ al examinar genes diferentes, sin que sea posible determinar con exactitud un gen único, lo que sugiere que son diferentes genes y que pueden actuar en diferentes etapas del desarrollo embrionario. ${ }^{5}$

La parte ambiental también ha sido estudiada, con énfasis en elementos tóxicos que puedan alterar el correcto desarrollo embriológico y afectar la migración celular o impedir los procesos de fusión. ${ }^{3}$ Los elementos ambientales más estudiados han sido el uso de pesticidas, fungicidas, el consumo de cigarrillo, alcohol y ácido fólico durante el embarazo que muestran asociaciones de riesgo de acuerdo con el tipo de estudio realizado y la población afectada. ${ }^{10}$ El nororiente colombiano, por ser una zona con prevalencia de esta malformación mayor a lo reportado en Colombia y otras naciones, ${ }^{2}$ se convierte en una zona de interés para realizar estudios que muestren cómo factores como el cigarrillo y el alcohol durante los tres primeros meses de embarazo pueden ser factores de riesgo para el labio y paladar hendido no sindrómico e hipodoncia.

\section{MATERIALES Y MÉTODOS}

Se realizó un estudio de corte transversal. La muestra estuvo constituida por los pacientes que asistieron a la convocatoria de Operación Sonrisa año 2001 en el Hospital Militar de Bogotá y en el Hospital Universitario Ramón González Valencia de la ciudad de Bucaramanga. Se incluyeron los pacientes con labio y/o paladar hendido no sindrómico desde los 4 años hasta los 18 años de edad que asistieron acompañados de su madre. Se excluyeron del estudio los pacientes sindrómicos, previa valoración por el Departamento de Genética.

Se analizaron las siguientes variables:

- Variables sociodemográficas: género, edad, residencia antes del embarazo, residencia durante el primer trimestre del embarazo y estrato socioeconómico.

- Antecedentes sobre el consumo de cigarrillo y alcohol en la madre: si ha fumado alguna vez en su vida, edad en la que empezó a fumar, aún fuma en la actualidad, fumó durante los tres primeros meses de embarazo, número de cigarrillos que fumaba en un día, hace cuánto dejó de fumar, años que duró fumando, convivencia con fumadores durante el primer trimestre del embarazo, hábito de tomar, tipo de bebida que ingierió, frecuencia con la que tomaba bebidas alcohólicas.

- Antecedentes y variables odontológicas: tipo de hendidura, lado de la hendidura, familiares con labio y/o paladar hendido, número de familiares con labio y/o paladar hendido, presencia de hipodoncia, hipodoncia de laterales, lado de la hipodoncia de laterales, hipodoncia de premolares, lado de la hipodoncia de premolares.

Los pacientes se atendieron según la numeración y orden dado por la organización del evento. Al acompañante de cada paciente se le explicaba el objeto de la investigación y se le solicitaba firmar un Consentimiento Informado que permitiera recoger la información y tomar una radiografia panorámica. Una vez el paciente accedía libremente a participar en el estudio se registraba la información y se entregaba la orden de la radiografia. Además se diligenció la historia clínica completa y el formulario ECLAM con anexo sobre antecedentes de cigarrillo y alcohol. La información se digitó en una base de datos creada en Excel. Para su análisis estadístico se utilizó el programa estadístico Stata I/C versión 9.0. Se realizó un análisis descriptivo de las variables sociodemográficas de la población de estudio, de acuerdo con su naturaleza y distribución. Se obtuvieron proporciones para las cualitativas y medidas de tendencia central para las cuantitativas. La prueba de $\mathrm{Chi}^{2}$ y Test exacto de Fisher fueron utilizadas para analizar las variables cua- 
litativas y la t de Student o U. de Mann Whitney para las cuantitativas. Una regresión logística determinó la posible asociación entre las variables consumo de cigarrillo y alcohol con la presencia de labio y/o paladar hendido no sindrómico. Se consideró un valor alpha igual a 0,05 .

\section{Criterios éticos}

La resolución 008430 de 1993 del Ministerio de Salud de la República de Colombia en la cual se establecen las normas científicas y administrativas de la investigación en salud, en sus atribuciones legales conferidas por el decreto 2164 de 1992 y la ley 10 de 1990, resuelve en el Título II Capítulo I de los aspectos éticos de la investigación en seres humanos. Este trabajo cumplió con los requisitos de los artículos donde prevalece el criterio de respeto a la dignidad y protección de los derechos y el bienestar de los pacientes de la muestra para el desarrollo de la investigación. Además, la investigación fue realizada por profesionales con conocimiento y experiencia para evitar cualquier daño en la integridad de éstos. Esta investigación se clasificó en la categoría con riesgo mayor que el mínimo por la radiación a la que fueron sometidos todos los pacientes. Con relación al Capítulo III donde se menciona la investigación en menores de edad o discapacitados, se cumplió con lo citado en los Artículos 23 y 24, ya que se aseguró que este tipo de investigaciones habían sido realizadas anteriormente en condiciones semejantes. Adicionalmente, se cumple con el Artí- culo 28 en su parágrafo segundo, donde se admiten investigaciones, según el riesgo y el beneficio a los pacientes, pues esta investigación buscó la prevención de los factores externos como el alcohol y el cigarrillo en posibles casos de labio y paladar hendido y la presencia de hipodoncia para lograr el bienestar de la comunidad y bajar el índice de malformaciones faciales en nuestra población.

\section{RESULTADOS}

En total se evaluaron 79 pacientes, de los cuales 37 $(46,84 \%)$ eran del género masculino, el promedio de edad fue 9,9 \pm 4,2 años. Estos pacientes provenían de diferentes municipios del país, principalmente de los departamentos de Boyacá, Santander, Norte de Santander, Cundinamarca, Caldas y Arauca. Las condiciones socioeconómicas fueron diversas, estrato 1 (33 pacientes, 41,77\%), estrato 2 (35 pacientes, $44,30 \%$ ), estrato 3 (10 pacientes, $12,66 \%$ ), estrato 4 (1 paciente, $1,27 \%$ ), con una edad promedio de la madre al embarazo de 28,8 \pm 6,3 años.

La Tabla 1 describe las variables relacionadas con la presencia de la anomalía en la muestra de estudio. La Tabla 2 presenta los antecedentes de consumo de cigarrillo en las madres de niños con labio y/o paladar hendido. En la Tabla 3 se aprecian los antecedentes de consumo de alcohol en las madres y la probabilidad de que se presente la anomalía según el hábito de fumar y el consumo de cigarrillo se evidencia en la Tabla 4.

Tabla 1. Distribución de frecuencias de las variables relacionadas con la presencia de labio y/o paladar hendido

\begin{tabular}{lc}
\hline \multicolumn{1}{c}{ Variables } & $\mathbf{n}(\%)$ \\
\hline Familiares con labio y paladar hendido & $39(49)$ \\
Pacientes con labio y paladar hendido & $76(96)$ \\
Lado derecho & $47(59)$ \\
Lado izquierdo & $21(27)$ \\
Bilateral & $11(14)$ \\
Pacientes con fisura labial & $3(4)$ \\
Pacientes con fisura palatina & -- \\
Pacientes con hipodoncia & $67(85)$ \\
Pacientes con hipodoncia incisivo lateral superior & $64(81)$ \\
Lado derecho hipodoncia incisivo lateral superior & $47(60)$ \\
Lado izquierdo hipodoncia incisivo lateral superior & $20(25)$ \\
Bilateral & $12(15)$ \\
Hipodoncia de premolares & $5(7)$ \\
\hline
\end{tabular}


Tabla 2. Antecedentes de consumo de cigarrillo en las madres evaluadas

\begin{tabular}{lcc}
\hline \multicolumn{1}{c}{ Factor de riesgo } & Frecuencia & Rango \\
\hline Antecedentes de fumadoras & $27(35)$ & \\
Fumadoras actuales & $18(23)$ & \\
Fumadoras tres primeros meses de embarazo & $22(28)$ & \\
Convivencia con fumadores & $41(52)$ & \\
& Promedio \pm D. E. & $12-20$ \\
Edad inicio de fumadoras & $16 \pm 2,2$ & $3-15$ \\
Número de cigarrillos diarios & $6 \pm 3,5$ & $1-21$ \\
Años que fumó & $11,3 \pm 9,6$ & \\
\hline
\end{tabular}

Tabla 3. Antecedentes de consumo de alcohol

\begin{tabular}{|c|c|}
\hline Variables & $\begin{array}{c}\text { Frecuencia } \\
\text { n (\%) }\end{array}$ \\
\hline Antecedentes de Alcohol & $34(43)$ \\
\hline Antecedentes alcohólicos durante los tres primeros meses de embarazo & $16(20)$ \\
\hline $\begin{array}{l}\text { Número de tragos consumidos } \\
\text { Uno a cinco tragos }\end{array}$ & $6(7,6)$ \\
\hline Seis a diez tragos & $7(8,9)$ \\
\hline Once a quince tragos & $3(3,8)$ \\
\hline
\end{tabular}

Tabla 4. Factor de riesgo del cigarrillo y alcohol

\begin{tabular}{lcc}
\hline \multicolumn{1}{c}{ Variable } & OR & IC 95\% \\
\hline Antecedentes de fumadoras & 1,06 & $0,91-12,26$ \\
Edad inicio de fumar & 1,01 & $0,87-1,18$ \\
Cantidad de alcohol ingerido & 1,50 & $0,13-17,66$ \\
\hline
\end{tabular}

Al resumir los hallazgos se puede concluir que la hendidura labio palatina fue la más frecuente, se presentó en 76 pacientes que corresponde al 96\% al ser el lado derecho el más afectado en 47 (59\%) pacientes (59\%), el izquierdo en 21 (27\%) pacientes y bilateral en $11(14 \%)$ Esto se relaciona con el lado en que se evidenció la hipodoncia debido a que se presentó hipodoncia derecha en 47 (59\%) pacientes, izquierda en 20 (25\%) y bilateral en $12(16 \%)$. Se observó hipodoncia en 67 (85\%) pacientes del grupo de estudio al ser la hipodoncia del incisivo lateral superior la más frecuente, $64(81 \%)$ pacientes y la hipodoncia de premolares en cinco (7\%) pacientes (7\%).

Cuando se evaluó el cigarrillo y el alcohol como factor de riesgo para tener hijos/as con labio y/o paladar no sindrómico, no se encontró una relación estadísticamente significativa, pero si se observó una relación positiva ya que 27 (35\%) de las madres presentaron antecedentes como fumadoras con una edad promedio de inicio de fumar $16 \pm 2,2$ años. Veintidós (28\%) madres reportaron haber fumado durante los tres primeros meses de embarazo con un promedio de cigarrillos de $6 \pm 3,5$ diarios. Esas madres llevaban fumando un promedio de 11,3 \pm 9,6 años. Al establecer factores de riesgo se encontró que variables como antecedentes de fumadora presentó un OR de 1,06 [IC 95\%: 0,91-12,26] y la edad de inicio de fumar un OR de 1,01 [IC 95\%:0,87 $-1.18]$.

Adicionalmente, se encontró que 34 (43\%) madres reportaron estar acostumbradas a tomar bebidas alcohólicas frecuentemente y $16(20 \%)$ de las 79 madres encuestadas tomaron alcohol durante los tres primeros meses de embarazo. Al establecer factores 
de riesgo sobre la cantidad de alcohol consumido durante los tres primeros meses de embarazo y la presencia de hendiduras orales se encontró un OR de 1,5 [IC 95\%: 1,33 - 17,6].

\section{DISCUSIÓN}

Otros estudios también han reportado que el tipo de hendidura más frecuente ha sido la labio palatina. Éricson y colaboradores, en 1979, encontraron que 51 (75\%) pacientes presentaron este tipo de hendidura. En 1991, Shaw y Lammer evaluaron un grupo de 731 personas, 447 (61\%) presentaban labio y paladar hendido. ${ }^{11}$ Shapira y colaboradores (2000) estudiaron a 238 pacientes, $198(66,4 \%)$ tenían labio y paladar hendido. ${ }^{12}$ En dos centros médicos de la ciudad de Bucaramanga, entre los años 2007 y 2009 se encontró un 55,6\% con labio y paladar hendido y $40,7 \%$ con labio o paladar aislados. ${ }^{2}$ Para el presente estudio de 79 pacientes se encontraron 76 (96\%) con labio y paladar hendido.

Los resultados sobre hipodoncia sugieren que ésta se presenta en alta frecuencia en pacientes con labio y paladar hendido. El presente estudio mostró una frecuencia de hipodoncia del $85 \%$ distribuida en un $81 \%$ de hipodoncia de laterales y un $7 \%$ de hipodoncia de premolares. Shapira y colaboradores encontraron un $74 \%$ de hipodoncia de laterales y un $18 \%$ de premolares. ${ }^{12}$ Se han reportado cifras de hipodoncia de laterales en la población normal del 2,2\% y en pacientes con labio y paladar del $56,9 \% .{ }^{13,14} \mathrm{En}$ un estudio realizado en Colombia, se buscó establecer el patrón de herencia de agenesias dentales en siete grupos familiares, se encontró que los dientes ausentes con mayor frecuencia fueron los laterales superiores $(46,1 \%)$ seguido por los segundos premolares inferiores $(7,69 \%) .{ }^{15}$ Los resultados del presente estudio mostraron hipodoncia de premolares del $7 \%$ en pacientes con labio y paladar hendido. Estos datos coinciden con resultados publicados en pacientes con hendiduras que han sido del 3,4\% y $6,6 \%$, aunque el estudio de Shapira y colaboradores encontró un $18 \%$ de hipodoncia de premolares, en un grupo con mayor proporción de labio y paladar hendido. ${ }^{12}$ También, se ha asociado la hipodoncia con gran cantidad de síndromes craneofaciales dentro de los que están la secuencia de Pierre Robin (69\%), síndrome de Van der Woude (70\%), diferentes formas de displasia ectodérmica y otros síndromes craneofaciales relacionados con hendiduras labiopalatinas. ${ }^{16}$

La alta frecuencia de hipodoncia en pacientes con labio y paladar sugiere la relación entre los mecanismos de formación de fisuras orales y los procesos de odontogénesis. Los dos procesos dependen de la gobernabilidad de la migración de las células de la cresta neural y de las interacciones epitelio mesénquima. ${ }^{17,18}$ Además, se ha mostrado que pueden tener la misma influencia genética: Satokata y Maas, utilizaron ratones transgénicos de MSX1 no funcional, género anodoncia y paladar hendido, para mostrar cómo el mismo grupo de genes que intervienen en la formación de la cara, están relacionados con la formación dental, una de las explicaciones de este desorden facial, es que la expresión del MSX1 en las células mesénquimas derivadas de la cresta neural se hallan en los procesos faciales y dentales. ${ }^{19}$ Thesleff señaló que los genes homeóticos, con sus factores de transcripción y de crecimiento, están implicados en la regulación de la morfogénesis y desarrollo craneofacial y dental del ser humano, mostrando como algunas proteínas como la proteína morfogénetica del hueso (BMP4), interviene en la regulación de la formación de estructuras esqueléticas, además de actuar como señal inductora epitelial en el desarrollo de los dientes al regular la expresión génica en el mesénquima dental, el cual incluye la expresión del MSX1, importante para la iniciación de dichas estructuras..$^{20,21}$

Otro estudio que resalta la evidencia de la base genética para hendiduras orales e hipodoncia es el estudio de Vastardis y colaboradores quienes localizaron la mutación del gen MSX1 en el cromosoma 4 (4p16) en todos los miembros afectados de una familia con hipodoncia de segundos premolares. ${ }^{22}$ La inactivación de MSX1 y PAX9 en ratones transgénicos causa la detención del desarrollo dental en el estado de campana y malformaciones como el paladar hendido. ${ }^{23}$ En humanos, la inactivación de una copia de los genes causa defectos dentales o defectos dentales y hendiduras en el caso mutación MSX1. ${ }^{24,25}$

Estos datos son de interés ya que establecen la asociación entre hendiduras orales e hipodoncias y en especial con el labio y paladar hendido. Se muestra en este estudio una relación directa entre lado de la hendidura y lado en que se presenta la hipodoncia, el $47 \%$ de hendiduras se presentó en el lado derecho y el $47 \%$ hipodoncia de laterales se presentó en el mismo lado. Lo que relaciona a nivel biológico que la formación de la fisura tiene relación con la presencia de la hipodoncia.

Según el estudio del árbol genealógico de la muestra, 39 pacientes (49\%) reportaron tener antecedes de labio y/o paladar hendido que moueostra un patrón poligénico por encontrarse la presencia de hendidura labio y/o palatina en varias líneas generacionales, tanto por herencia materna como paterna. Es importante resaltar que no se encontró un patrón autosómico dominante o recesivo lo cual puede de- 
mostrar que también intervienen factores ambientales como pueden ser el alcohol o el cigarrillo, esto también lo demuestran estudios previos donde relacionan factores externos del medio ambiente que intervienen directamente sobre los genes que están implicados en el labio y paladar hendido. ${ }^{26}$

Al comparar los resultados de consumo de cigarrillo con presencia de hendiduras orales con resultados de otros estudios, se observó que los análisis sugieren una pequeña asociación estadística significativa entre fumar cigarrillo durante el primer trimestre de embarazo y el incremento del riesgo de tener niños con hendiduras. Los resultados del meta análisis realizado por Wyszynski y colaboradores (1997) sugieren un factor de riesgo promedio entre los 11 estudios revisados con criterios satisfactorios para la hendidura labio-palatina de [OR:1,32 IC 95\%:1,10 -1,62] que indica un pequeño incremento de riesgo a tener niños con hendidura. ${ }^{27}$ Esta asociación se incrementa al aumentar el número de cigarrillos por día como se observa en otra investigación en la que se incrementaba el riesgo cuando la madre fumaba de 1 a 9 cigarrillos o más de 20 cigarrillos. ${ }^{10,28}$

El consumo de alcohol, en la madre embarazada ha mostrado un potencial teratogénico. Estudios de laboratorio con ratones con síndrome de alcoholismo fetal han mostrado una forma leve de holoprosencefalia, deficiencia en la línea media del plato neural anterior que ocasiona que las placodas olfatorias tengan una alteración y cambios secundarios como fisuras orales. Cultivos de neuronas de estos ratones han mostrado alteraciones estructurales y funcionales de astrócitos que ocasionan disturbios en moléculas receptoras de matriz extracelular que están relacionadas con la migración de las células de la cresta neural. ${ }^{29,30}$

Los datos del presente estudio mostraron que un $20 \%$ de las madres consumieron alcohol durante el embarazo y aunque no se observó una relación de riesgo estadísticamente significativa sí se encontró una relación positiva que coincide con otros estudios publicados. Munger y colaboradores, en un estudio de casos y controles, reportaron cómo se incrementa la probabilidad de presentar labio y paladar a medida que aumenta el consumo, observaron además, mayor riesgo para labio y paladar que para paladar. ${ }^{31}$

El presente estudio también mostró asociación entre la cantidad de alcohol ingerido y la presencia de hendiduras orales [OR:1,5 IC 95\% 0,13-17,66]. Shaw y Lammer mostraron que madres que reportaron más de cinco tragos por ocasión comparadas con las que no tomaron, sí mostraron un incremento en el riesgo de que sus hijos nacieran con labio hen- dido, concluyeron que un alto consumo incrementa riesgos de hendidura y en especial de labio., ${ }^{11,32}$ Lorente y colaboradores encontraron un riesgo incrementado de paladar hendido con el consumo de alcohol [OR: 2,28 IC 95\%: 1,07 - 3,04] y establecieron dos categorías para el consumo de alcohol, más de 70 gramos y menos de 70 gramos. ${ }^{33} \mathrm{~A}$ medida que aumenta la dosis aumenta el riesgo.

Es necesario tener en cuenta que aunque las variables ambientales, como el hábito de fumar y el consumo de alcohol pueden aumentar el riesgo de hendiduras, no son los únicos factores para tener en cuenta, la etiología es poligénica y multifactorial, y estos factores ambientales pueden modular la respuesta genética. Está postulado que la interacción del cigarrillo con una variante alélica del TGFB3 y del alcohol con el MSX1 son factores importantes asociados al desarrollo del labio y paladar hendido. Romitti y colaboradores, en un estudio de casos y controles examinaron las variantes alélicas de tres genes: (TGFA), (TGFB3) y (MSX1) y su relación con la exposición durante el embarazo al cigarrillo y alcohol, encontraron riesgo de presentar paladar hendido en los hijos/as de las madres que fumaban más de 10 cigarrillos día con variantes alélicas TGFB3 o MSX1 en comparación con el riesgo de consumo de más de cuatro tragos por mes para labio y paladar y variante alélica del MSX1. ${ }^{34}$ Por lo tanto, se ha sugerido que el desarrollo del labio y paladar hendido puede estar influenciado independientemente por la exposición materna, pero más significativamente por la interacción de exposición a tales factores y variantes alélicas específicas. ${ }^{34-36}$

Es importante complementar este trabajo con datos de estudios genéticos para un mejor entendimiento de la información puesto que los reportes en labio y paladar e hipodoncia muestran una influencia genética en su etiología. Posteriores estudios con diseños de casos y controles y mayor tamaño de la muestra aplicada a nuestra población permitirán continuar con esta Línea de Investigación.

\section{Conclusiones}

No se encontró una relación estadísticamente significativa entre el consumo de cigarrillo y alcohol como factor de riesgo en labio y paladar hendido pero sí hubo una relación positiva entre las variables antes mencionadas para tener hijos con labio y paladar hendido no sindrómico.

En los pacientes con labio y paladar hendido se encontró hipodoncia en el $85 \%$ de los casos, la hipodoncia del incisivo lateral superior es la más frecuente en el $81 \%$ de los casos, seguido de la hipodoncia de premolares en el $7 \%$ de los casos. 


\section{BIBLIOGRAFÍA}

1. Serrano CA, Ruiz JM, Quiceno LF, Rodríguez MJ. Labio y/o paladar hendido: una revisión. Ustasalud 2009; 8: 44 - 51.

2. Rodríguez MJ, Serrano CA, Ruiz JM, Quiceno LF. Epidemiology of oral clefts in two colombian hospitals. J Dent Res 91: abstract 1301, 2012.

3. Murray JC. Gene/environment causes of cleft lip and/or palate. Clin Genetic 2002; 61: 248 - 256.

4. Thorogood P, Ferreti P. Heads and tales: recent advances in craniofacial development. Br Dent J 1992; 173: 301 - 306.

5. Parada $C$, Bayona F. Palatogénesis y hendiduras palatinas. Acta Biológica Colombiana 2004; 9: 15 - 22.

6. Moore K. Persaud T. Embriología Clínica. Quinta Edición, Editorial interamericana, México; 1997.

7. Gorlin RJ. Sindromes of the head and neck, Oxford University Press. 1990.

8. Vieira A, Taucher S. Análisis mutacional del gen homeobox MSX1 en chilenos con fisuras orales. Rev Med Chile 2004; 132: 816 - 822.

9. Otero L, Gutiérrez S. Association of MSX1 with nonsyndromic cleft and lip palate in a Colombian population. Cleft palate Craniofac 2007; 44: 653 - 656.

10. Goncalves LI, Koifman S. Oral clefts, consanguinity, parental tobacco and alcohol use: a case control study in Rio de Janeiro, Brazil. Braz Oral Res 2009; 23: 31- 37.

11. Shaw GM, Lammer EJ. Maternal periconceptional alcohol consumption and risk for orofacial clefts. J Pediatr 1999; 134: 298 - 303.

12. Shapira Y, Lubit E, Kuftinec MM. Hypodontia in children with various types of clefts. Angle Orthod 2000; 70: 16 - 22.

13. Graber LW.Congenital absence of teeth: a review with emphasis on inheritance patterns J Am Dent Assoc 1978; 96: 266 - 275

14. Suárez BK, Spence MA The genetics of hypodontia. J Dent Res 1974; 53: 781 - 785.

15. Quintero M, Berrocal M. La agenesia dental y su mecanismo de herencia en siete grupos familiares. Universitas Odontológica 2002; 49: 27 - 36.

16. Sperber GH. First year of life: prenatal craniofacial development. Cleft Palate Craniofac J 1992; 29: 109 - 113.

17. Johnston MC, Bronsky PT. Prenatal craniofacial development: New insights on normal and abnormal mechanisms. Crit Rev Oral Biolo Med 1995; 6: 25 - 79.

18. Thomas T, Kurihara H, Yamagishi H, Kurihara Y, Yazaki Y, Olson EN, Srivastava D. A signaling cascade involving endothelin-1, dHAND and msx1 regulates development of neural-crest-derived branchial arch mesenchyme. Development 1998; 125: 3005 - 3012.

19. Satokata I, Maas R. MSX1 deficient mice exhibit cleft palate and abnormalities of craniofacial and tooth development. Nat Genet 1994; 6: 348 - 356.

20. Thesleff I. Homeobox genes and growth factors in regulation of craniofacial and tooth morphogenesis. Acta Odontol Scand 1995; 53: 129 - 134.

21. Nieminen P, Arte S, Pirinen S, Peltonen L, Thesleff I. Gene defect in hypodontia: exclusion of MSX1 and MSX2 as candidate genes. Hum Genet 1995; 96: 305 - 308.

22. Vastardis H, Karimbux N, Guthua SW, Seidman JG, Seidman CE. A human MSX1 homeodomain missense muta- tion causes selective tooth agenesis. Nat Genet 1996; 13 : $415-423$.

23. Martin JF, bradley A, Olson EN. The paired-like homeo box gene Mhox is required for early events of skeletogenesis in multiple lineages. Genes Dev 1995; 10: 1237 - 1249.

24. Stanford LP, Ormsby I, Gittenberger-DE Groot AC, Sariola H, Friedman R, Boivin GP, Cardell EL, Doetschman T. TGF beta2 knockout mice have multiple developmental defects that are non-overlapping with other TGFbeta knockout phenotypes. Development 1997; 124: 2659 - 2670.

25. Arte S. Phenotipic and genotipic features of familial hypodontia. Institute of Dentistry, Department of Pedodontics and Orthodontics. University of Helsinki, Finland Academic Dissertation 2001. URL disponible en: http://ethesis. helsinki. fi/julkaisut/laa/hamma/vk/arte/phenotyp.pdf

26. Young DL, Schneider RA, Hu D, Helms JA. Genetic and teratogenic approaches to craniofacial development. Crit Rev Oral Biol Med 2000; 11: 304 - 317.

27. Wyszynski DF, Wu T. Use birth certificate data to estimate the risk of maternal cigarette smoking for oral clefting. Cleft Palate Craniofac J 2002; 39: 188 - 192.

28. Wang X, Tager IB, Van Vunakis H, Speizer FE, Hanrahan JP. Maternal smoking during pregnancy, urine cotinine concentrations, and birth outcomes. A prospective cohort study. Int J Epidemiol 1997; 26: 978 - 988.

29. Guerri C. Teratogenic effects of alcohol. Arch Tioxicol 1995; 18: 71 - 80.

30. Montoliu C, Valles S, Renau-Piqueras J, Guerri C. Ethanol induced oxygen radical formation and lipid peroxidation in rat brain: effect of chronic alcohol consumption. J Neurochem 1994; 63: 1855 - 1862.

31. Munger RG, Romitti PA, Daarck-Hirsch S, Burns TL, Murray JC, Hanson J. Maternal alcohol use and risk of orofacial cleft birth defects. Teratology 1996; 54: 27 - 33.

32. Shaw GM. Maternal smoking and cleft palate. Teratology. 1991; 43: $225-228$.

33. Lorente C, Cordier S, Goujard J, Ayme S, Bianchi F, Calzolari E, De Walle HE, Knill-Jones R. Tobacco and alcohol use during pregnancy and risk of oral clefts. Occupational Exposure and Congenital Malformation Working Group. Am J Public Health 2000; 90: 415 - 419.

34. Rommitti PA, Lidral AC, Munger RG, Daack-Hirsch S, Burns TL, Murray JC Candidate genes for nonsyndromic cleft lip and palate and maternal cigarette smoking and alcohol consumption: evaluation of genotype-environment interactions from a population based case-control. Teratology 1999; 59: 39 - 50.

35. Martin JF, Bradley A, Olson EN. The paired-like homeo box gene Mhox is required for early events of skeletogenesis in multiple lineages. Genes Dev 1995; 10: 1237 - 1249.

36. Natsume N, Kawai T, Ogi N, Yoshida W. Maternal risk factors in cleft lip and palate: case control study. Br J Oral Maxillofac Surg 2000; 38: 23 - 35.

\section{Correos electrónicos de los autores:}

Ethman Ariel Torres Murillo: ethmant@yahoo.com Germán Gómez Lázaro: germangomez12@hotmail.com Zonia Pinzón Bayona: zoniapinzon@hotmail.com 